\title{
Effects of CCR5 59029G/A polymorphism on the risk to diabetic nephropathy
}

\author{
Mingfeng Cao ${ }^{1}$, Zhenhua Tian ${ }^{1}$, Lin Zhang ${ }^{1}$, Ruiting Liu ${ }^{1}$, Qingbo Guan ${ }^{1}$ and Jinjiao \\ Jiang ${ }^{1}$ \\ ${ }^{1}$ Department of Endocrinology, Shandong Provincial Hospital Affilated to Shandong University, Jinan 250100, Shandong, \\ China \\ Correspondance to: Jinjiao Jiang, email: akrryj46@163.com \\ Keywords: CCR5; chemokine; diabetic nephropathy; polymorphism; meta-analysis \\ Received: July 02, $2016 \quad$ Accepted: June 13, $2017 \quad$ Published: October 30, 2017 \\ Copyright: Cao et al. This is an open-access article distributed under the terms of the Creative Commons Attribution License 3.0 \\ (CC BY 3.0), which permits unrestricted use, distribution, and reproduction in any medium, provided the original author and source \\ are credited.
}

\section{ABSTRACT}

Background: Diabetic nephropathy (DN) causes high mortality in patients with diabetes mellitus and imposes heavy burden on individuals and society. In previous studies, various researches have investigated the association of DN with CCR5 59029G/A polymorphism, but relevant findings were controversial. Therefore, we performed this meta-analysis to obtain a conclusion on this issue.

Results: CCR5 59029G/A polymorphism showed significant risk-increasing effects on DN in all analyses under AA vs. GG, AA+GA vs. GG, AA vs. GG+GA, A vs. G and GA vs. GG model contrasts. Besides, a similar result was also obtained in Asian and type 2 diabetes mellitus groups under these five contrasts after subgroup analyses.

Methods: The relevant publications were searched from the electronic databases and other sources. The association intensity between CCR5 59029G/A polymorphism and DN susceptibility was measured using pooled odds ratios (ORs) with corresponding $95 \%$ confidence intervals ( $95 \%$ CIs). Inter-study heterogeneity was inspected with Q test, and sensitivity analysis was conducted to verify the stability of the final outcomes by removing one study each time in turn. Begg's funnel plot and Egger's test were utilized to examine publication bias among selected studies.

Conclusion: CCR5 59029G/A polymorphism is significantly related to enhanced susceptibility to DN, especially in Asian populations and people with type 2 diabetes mellitus.

\section{INTRODUCTION}

Diabetic nephropathy (DN), a common microvascular complication of diabetes, is a major cause of chronic kidney diseases, and results in high mortality in diabetic patients $[1,2]$. As a medical condition greatly jeopardizing peoples' health, DN is a leading cause of end-stage renal disease (ESRD), accounting for approximately half of patients receiving dialysis treatment [3-5]. DN can cause reduced filtration, albuminuria, and ultimately renal failure [6], seriously threatening patients' lives. During early stage, it can be detected through some changes in renal metabolism and structure [7]. The pathogenesis of DN is complex, and involves multiple pathways, including advanced glycation end products, oxidative stress, inflammatory cytokines and profibrotic factors [8]. There are some methods to detect early changes in diabetic disease, but effective therapeutic measures have not been developed yet [9]. Therefore, it is urgent to ascertain the pathophysiology mechanism of DN for effectively controlling over this disease [10].

Chemokines, inducible proinflammatory cytokines, are regarded as important determinants of early inflammatory responses [11]. The cysteine-cysteine (CC) motif chemokine receptor 5 (CCR5) is involved in immune system where $\mathrm{T}$ cells are attracted to specific tissues and organ targets [12], and is constitutively expressed on some immune cells, like $\mathrm{T}$ lymphocytes and macrophages [13]. Up-regulated by proinflammatory cytokines, CCR5 acts as a receptor for 
multiple chemokines, such as chemokine ligand 5 (CCL5), macrophage inflammatory protein-1a (MIP-1a), macrophage inflammatory protein-1b (MIP-1b), and monocyte chemoattractant protein-2 (MCP-2) [14-16]. The gene CCR5 is polymorphic and located within the gene cluster on chromosome 3p21.3-p24 [17]. The polymorphisms in this gene may affect the activity of the protein, thus contributing to multiple diseases, including HIV-1 infection [18], cancers [19], tuberculosis [20], atopic asthma [21] and DN [22].

Among the polymorphisms of CCR5 gene, 59029G/A is frequently-reported to be relevant to the susceptibility to DN, but no consensus has been achieved on this issue yet. To systematically explore the effects of CCR5 59029G/A polymorphism on susceptibility to $\mathrm{DN}$, we carried out this meta-analysis by pooling the previous findings.

\section{RESULTS}

\section{Study characteristics}

Initially, a total of 128 publications were retrieved through searching databases and other sources. In literature screening, 115 reports were excluded for duplicate (1), irrelevant title and abstracts (76), and other reasons (38). Among the 38 literature excluded by other reasons, 12 were for not related to CCR5 59029 polymorphism, 11 reports were without available data, 10 were reviews or comments, and the resting 5 article were published in other languages (Figure 1). Hence, 13 eligible articles were ultimately incorporated into this meta-analysis [22-34]. Among them, 3 were in Caucasian populations while the others were in Asian populations. Additionally, the quality assessment of these studies based on NOS showed that only the article of Pettigrew [28] had the relative poor quality with 4 scores and the quality the other included studies was good. Table 1 summarized the essential information of all included studies.

\section{Quantitative data synthesis}

As shown in Table 2, CCR5 59029G/A polymorphism dramatically increased the susceptibility to DN in total analysis under the five genetic comparisons of AA vs. GG, AA+GA vs. GG, AA vs. GG+GA, A vs. G and GA vs. GG $(\mathrm{OR}=2.46,95 \% \mathrm{CI}=1.68-3.63$ (Figure 2); $\mathrm{OR}=2.21,95 \%$ $\mathrm{CI}=1.60-3.05$ (Figure 3); $\mathrm{OR}=1.53,95 \% \mathrm{CI}=1.21-1.94$; $\mathrm{OR}=1.63$, 95\% CI=1.32-2.01; $\mathrm{OR}=1.98,95 \% \mathrm{CI}=1.47-$ 2.66). In addition, under the same five contrasts, a similar effect of the polymorphism was also observed in Asian (Figure 2) and type 2 diabetes mellitus (Figure 3) groups after subgroup analyses based on ethnicity and type of diabetes mellitus.

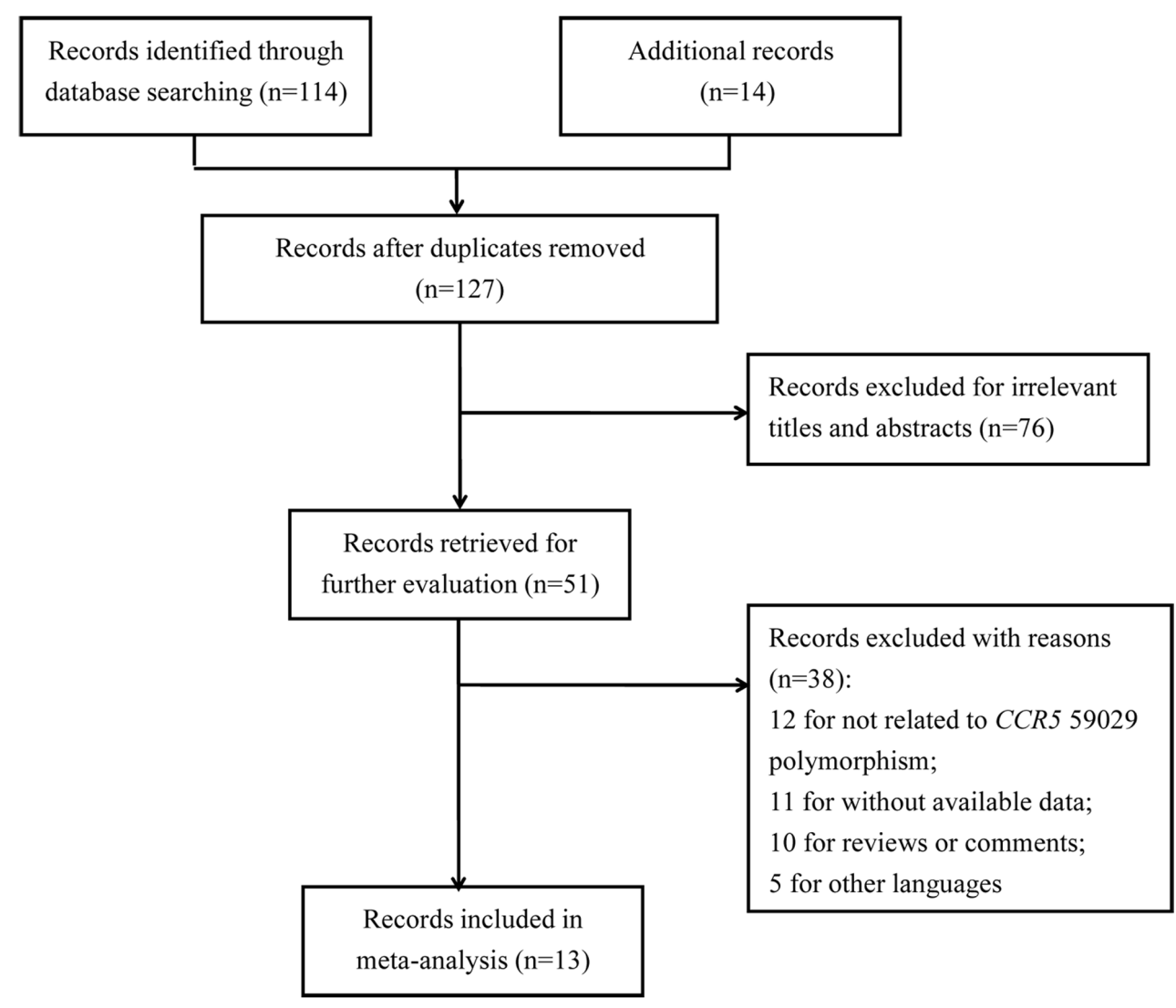

Figure 1: Flow diagram for the process of literature selecting. 
Table 1: Principal information of studies included in the meta-analysis

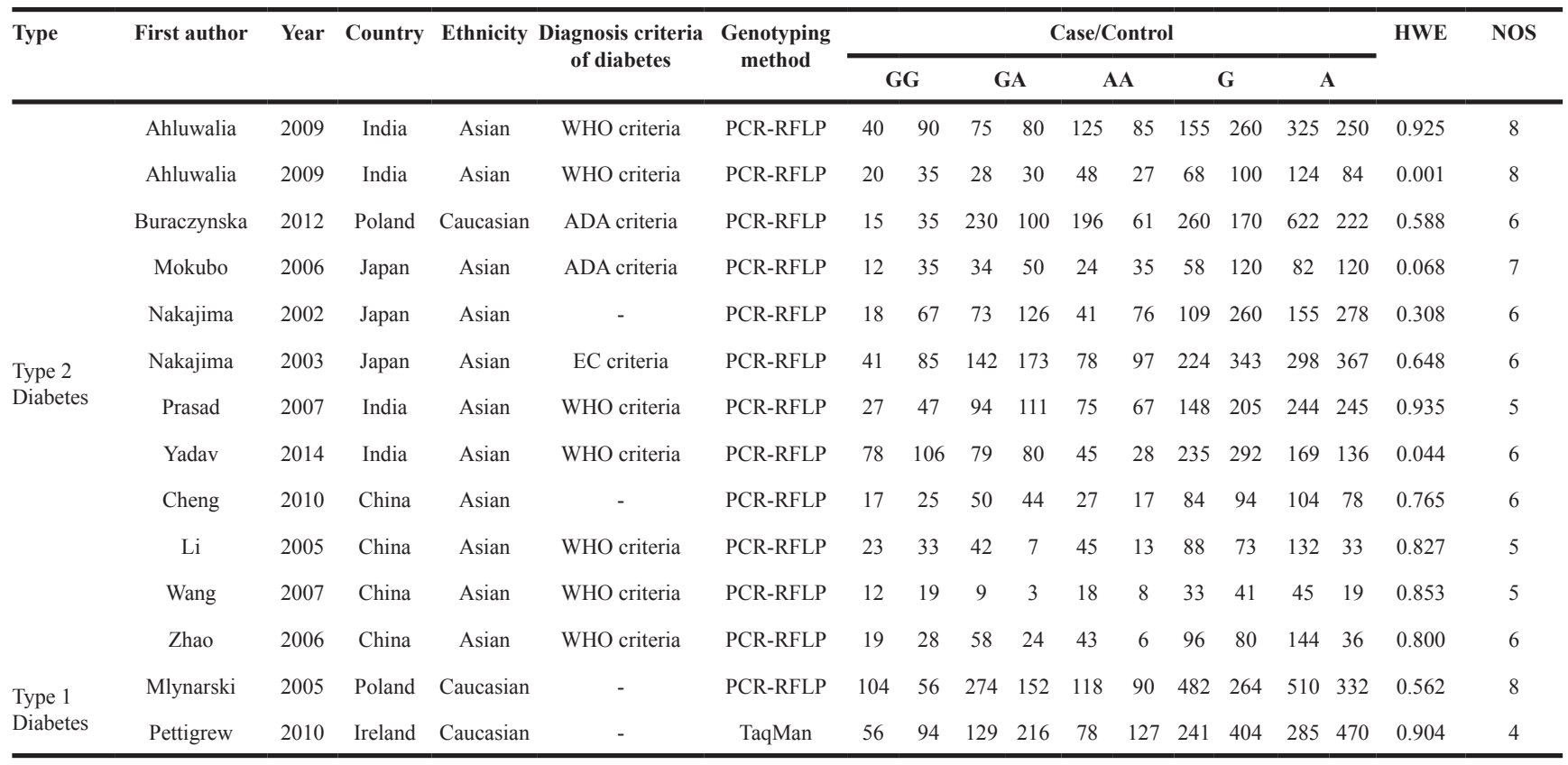

Notes: WHO: World Health Organization; ADA: American Diabetes Association; EC: Expert Committee; PCR-RFLP, polymerase chain reaction-restriction fragment length polymorphism; TaqMan, TaqManSNP; HWE, Hardy-Weinberg equilibrium; NOS: Newcastle-Ottawa Scale.

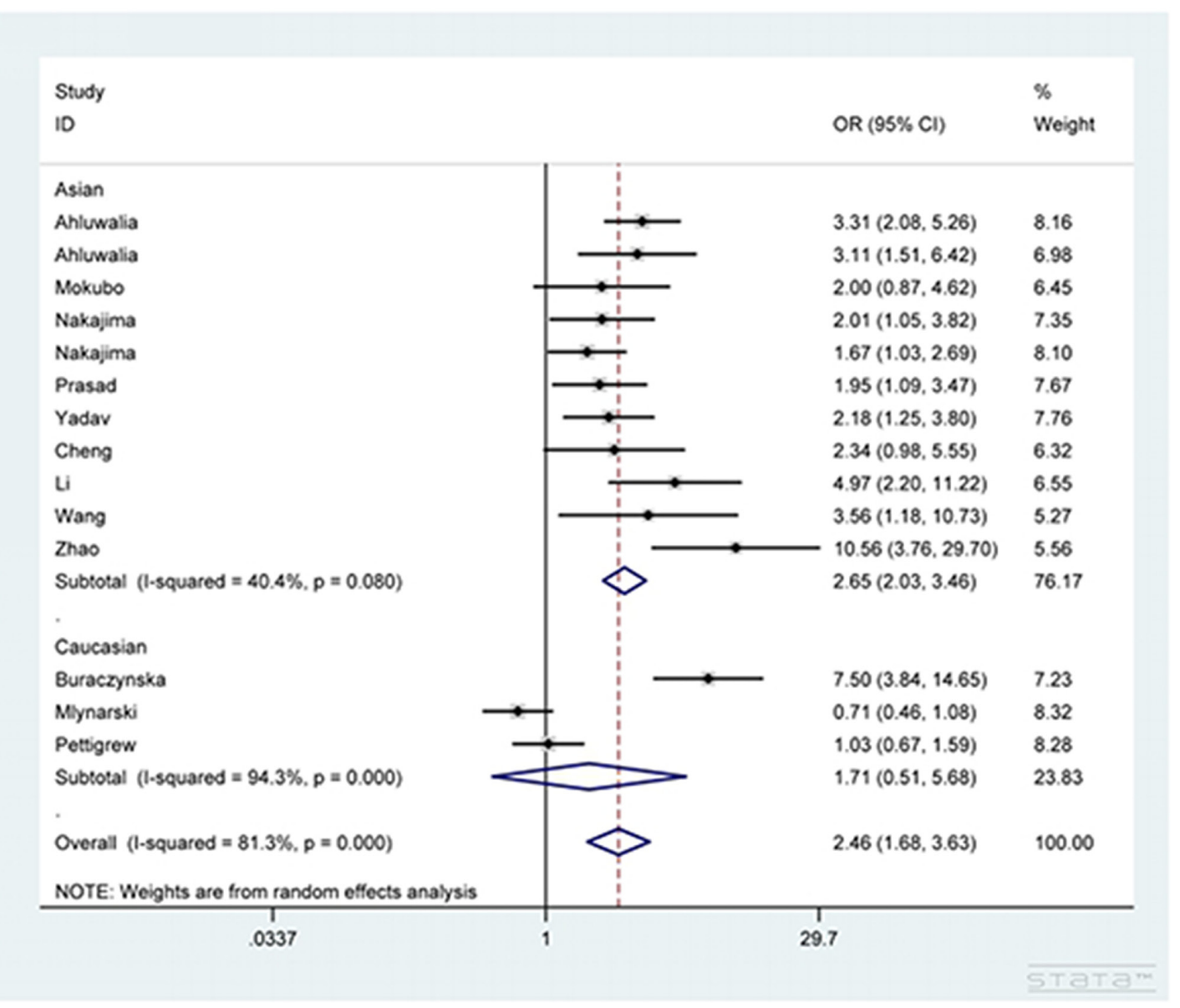

Figure 2: Forest plot for the association between CCR5 59029G/A polymorphism and diabetic nephropathy susceptibility under AA vs. GG contrast. 
Table 2: CCR5 59029G/A polymorphism and diabetic nephropathy susceptibility

\begin{tabular}{|c|c|c|c|c|c|c|c|c|c|c|c|}
\hline \multirow{3}{*}{$\begin{array}{l}\text { Group } \\
\text { Asian }\end{array}$} & \multirow{3}{*}{$\begin{array}{c}\begin{array}{c}\text { No. of } \\
\text { studies }\end{array} \\
11\end{array}$} & \multicolumn{10}{|c|}{ Odds ratio $(95 \%$ confidence interval) / $P$ value for heterogeneity } \\
\hline & & \multicolumn{2}{|c|}{ AA versus GG } & \multicolumn{2}{|c|}{$\mathbf{A A}+\mathbf{G A}$ versus $\mathbf{G G}$} & \multicolumn{2}{|c|}{ AA versus GG+GA } & \multicolumn{2}{|l|}{ A versus $G$} & \multicolumn{2}{|c|}{ GA versus GG } \\
\hline & & $2.65(2.03,3.46)$ & 0.080 & $2.35(1.82,3.03)$ & 0.019 & $1.70(1.37,2.12)$ & 0.055 & $1.80(1.47,2.20)$ & 0.000 & $2.04(1.57,2.64)$ & 0.057 \\
\hline Caucasian & 3 & $1.71(0.51,5.68)$ & 0.000 & $1.69(0.64,4.47)$ & 0.000 & $1.09(0.66,1.81)$ & 0.001 & $1.16(0.74,1.79)$ & 0.000 & $1.66(0.69,4.02)$ & 0.000 \\
\hline $\begin{array}{l}\text { Type } 2 \\
\text { Diabetes }\end{array}$ & 2 & $2.96(2.19,4.01)$ & 0.007 & $2.58(1.94,3.42)$ & 0.001 & $1.70(1.40,2.06)$ & 0.077 & $1.79(1.50,2.14)$ & 0.000 & $2.27(1.70,3.04)$ & 0.005 \\
\hline $\begin{array}{l}\text { Type } 1 \\
\text { Diabetes }\end{array}$ & 12 & $0.85(0.59,1.23)$ & 0.222 & $0.94(0.72,1.22)$ & 0.573 & $0.86(0.61,1.22)$ & 0.135 & $0.92(0.77,1.11)$ & 0.213 & $0.99(0.75,1.30)$ & 0.908 \\
\hline Total & 14 & $2.46(1.68,3.63)$ & 0.000 & $2.21(1.60,3.05)$ & 0.000 & $1.53(1.21,1.94)$ & 0.000 & $1.63(1.32,2.01)$ & 0.000 & $1.98(1.47,2.66)$ & 0.000 \\
\hline
\end{tabular}

\section{Heterogeneity test}

In total analysis, significant heterogeneity was detected under all five genetic models, so the randomeffects model was chosen for calculating pooled ORs. After stratified analyses by ethnicity and type of diabetes mellitus, the degree of heterogeneity significance was alleviated in some subgroups, indicating these two aspects might be related to the source of significant heterogeneity.

\section{Sensitivity analysis}

Sensitivity analysis was completed via deleting one single study each time and then re-calculating pooled ORs

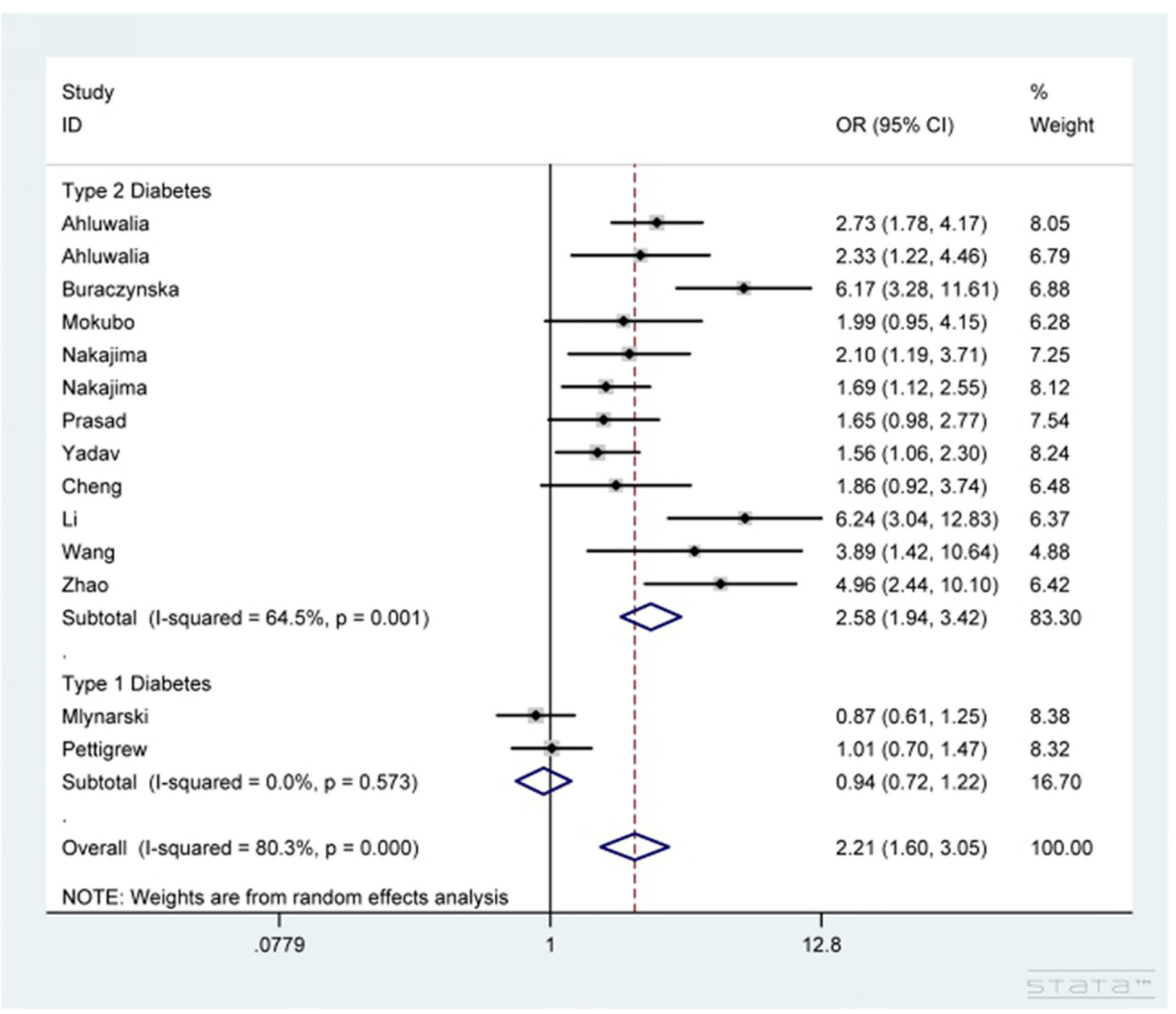

Figure 3: Forest plot for the association between CCR5 59029G/A polymorphism and diabetic nephropathy susceptibility under AA+GA vs. GG contrast after stratification analysis by type of diabetes mellitus. 
to observe alteration in final results. During this whole process, no material variation was detected after removing any one of the studies (Figure 4), showing the statistical robustness of our findings.

\section{Publication bias examination}

Publication bias across included studies was investigated with both Begg's funnel plot and Egger's test. As a result, the shape of funnel plots seemed asymmetric (Figure 5), implying the presence of significant publication bias. Furthermore, these results were statistically confirmed by evidence from Egger's test $(P=0.000)$. Such situation might be attributed to multiple aspects, such as studies with negative results more likely to be published in local rather than international journals, reports with null findings or small sample sizes having less chance to be published, as well as the interests of sponsors or beneficiaries. Non-parametric "trim and fill' method was used to adjust publication bias and the results showed that no trimming was performed, the data was unchanged (Figure 6). The results demonstrated that the asymmetry of the funnel was not caused by the publication bias.

\section{DISCUSSION}

As a serious complication of diabetes, DN is the leading cause of ESRD. Accompanied by high levels of

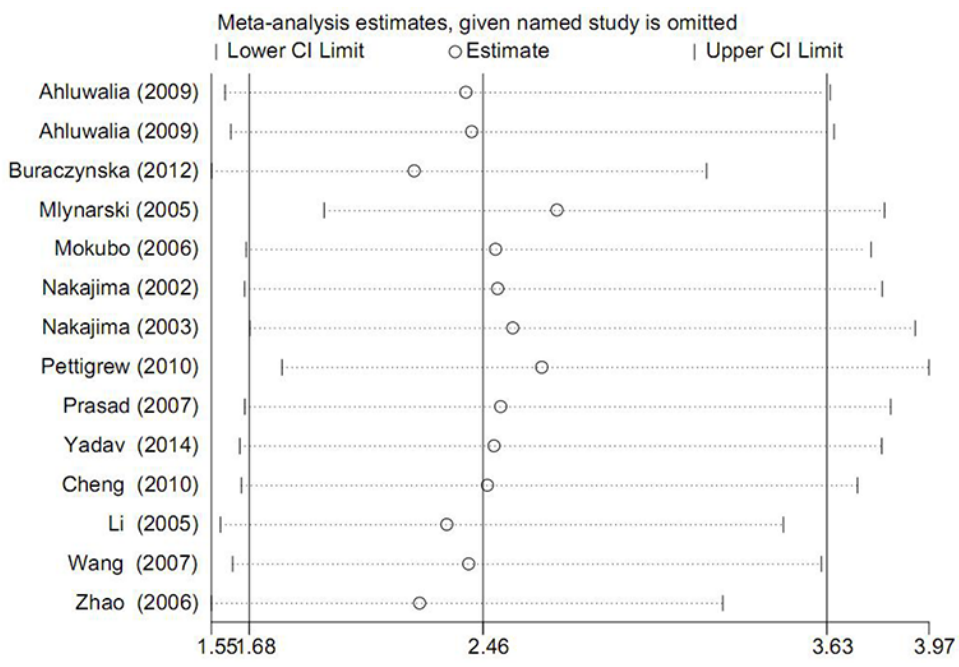

Figure 4: Forest plot for sensitivity analysis under AA vs. GG contrast.

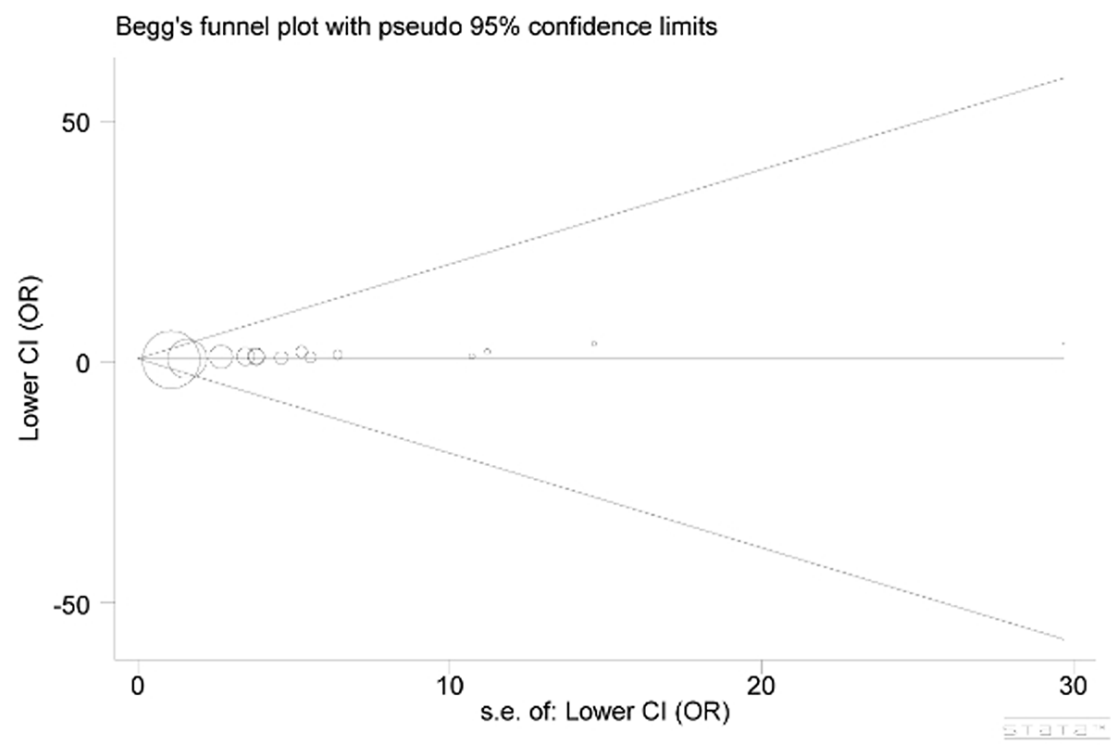

Figure 5: Begg's funnel plot for publication bias. 
pressure, sugar, lipids, protein and viscosity in blood as well as microcirculation disturbance, DN can result in the arteriosclerosis of kidney glomerulus, thus leading to edema and proteinuria. The incidence of DN is associated with many factors, such as high glucose, oxidative stress, metabolic disorder, and hemodynamics change. Besides, DN shows a familial aggregation tendency, indicating the important role of hereditary factors in its etiology. Nevertheless, the precise etiology of this disease still remains obscure at present. CCR5 is a G protein coupled receptor, and consists of 352 amino acids with a molecular weight of $40.6 \mathrm{KD}$. It is expressed on the surface of mononuclear macrophages, dendritic cells and memory $\mathrm{T}$ cells, and is constituted by 7 transmembrane domains, extracellular N-terminal, extracellular and intracellular domains, and intracellular C-terminal. As a $\beta$ chemokine factor, CCR5 plays vital roles in the chemotaxis, proliferation and immunoregulation of inflammatory cells. The polymorphism $59029 \mathrm{G} / \mathrm{A}$ in promoter regions of CCR5 gene can alter the activity of CCR5 protein, which has been reported to be correlated to the susceptibility of individuals to DN by various researchers.

For example, Ahluwalia et al. found a significantly higher frequency of the AA genotype of CCR 59029G/A polymorphism in patients with $\mathrm{DN}$ in both North and South Indians, showing the susceptible role of the genotype [22]. Additionally, in Caucasians of Polish origin, Buraczynska et al. found a risk-increasing effect of A allele on DN in patients with type 2 diabetic mellitus [23]. Besides, a similar influence of A allele and/or A+ genotype(s) of the polymorphism on susceptibility to DN was also replicated in several Chinese populations [3134]. In contrast, Mlynarski et al. showed that in type 1 diabetic patients, male carriers of $\mathrm{G}$ allele had a 1.9-fold higher risk of developing DN compared with non-carriers [24]. Moreover, the study by Pettigrew et al. revealed no significant correlation of the polymorphism with the risk of DN either [28]. There were no conclusions on this issue.

To obtain a more reliable conclusion, we performed this meta-analysis based on previously published studies, and the results manifested a significant risk-enhancing effect of the polymorphism on the risk of DN in total analysis as well as Asian and type 2 diabetes mellitus groups under the five genetic contrasts of AA vs. GG, $\mathrm{AA}+\mathrm{GA}$ vs. GG, AA vs. GG+GA, A vs. G and GA vs. GG.

There were some inevitable shortcomings in the present meta-analysis. First, the number of included studies was relatively small, which might affect the comprehensiveness of our findings. Meanwhile, the majority of included studies were based on Asian populations, which might introduce some selection bias. Second, the baseline characteristics like age and gender distribution, ethnicity, life styles, and family history in different studies might be statistically different,

Filled funnel plot with pseudo $95 \%$ confidence limits

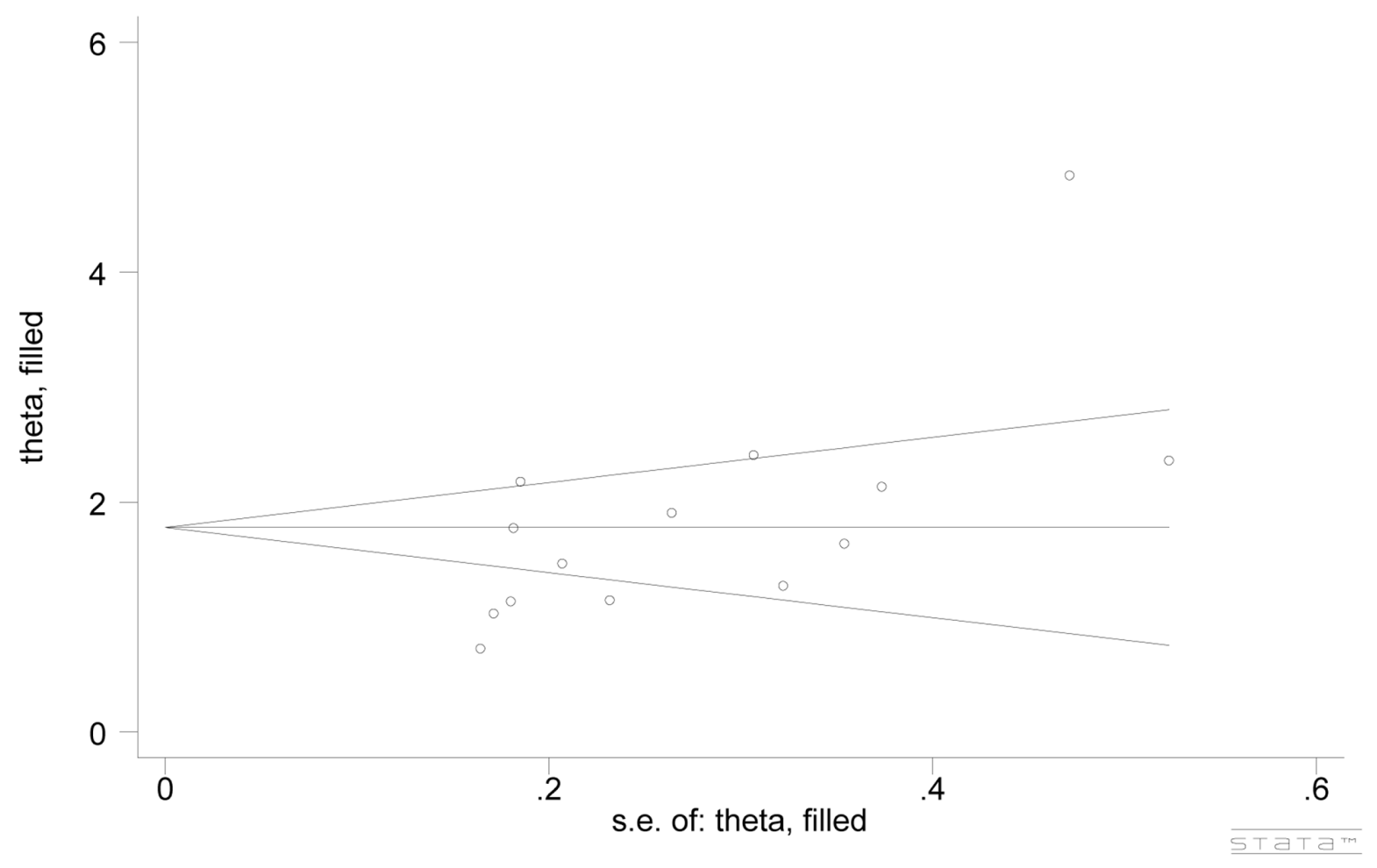

Figure 6: Begg's funnel plot adjusted by non-parametric "trim and fill' method. 
thus causing some clinical heterogeneity. Third, the accuracy of genotyping in the included studies could not be guaranteed. Among the 13 included studies, PCR-RFLP was the most frequently used method for genotyping. Compared with direct sequencing, PCRRFLP might exhibit low accuracy. Furthermore, the detailed information about source of control and the diagnostic criteria of DN was not available in some included studies. Last but not least, the potential joint effects of the studied polymorphism with other relevant factors were not explored in this study. Additionally, the shape of funnel plots seemed asymmetric in the current study, but the analysis performed using non-parametric 'trim and fill' method indicated that publication bias was not responsible for the asymmetry. The model used for analysis might also heterogeneity to the final results $[35,36]$. The multivariate model was needed to be constructed to estimate the genotype-disease associations. Further investigations will be required to address the above issues.

In conclusion, the results from the present meta-analysis provided additional evidences for the significant relationship of CCR5 59029G/A polymorphism with the susceptibility to DN. Considering the above-mentioned restrictions, our findings still need to be verified in future by studies with larger sample sizes and concerning potential genegene and gene-environment joint effects.

\section{MATERIALS AND METHODS}

\section{Literature searching}

The relevant publications were systemically searched from electronic databases of PubMed, EMBASE, BIOSIS and CNKI. Only the studies published in English or Chinese language were considered to be included. The combination of the following key terms were used for literature searching: "C-C chemokine receptor type 5" or "CCR5" or "CD195" or "CKR5", "diabetic nephropathy" or "DN", and "polymorphism" or "mutation" or "variant". Meanwhile, other sources were also checked for the same purpose. Besides, the references of relevant articles were also screened for additional reports.

\section{Inclusion and exclusion criteria}

All eligible articles must conform to the following criteria: (1) with a case-control design: the individuals in control group were Type 2 Diabetes or Type 1 Diabetes patients without nephropathy, while patients in case group were DN cases; (2) assessing the association between CCR5 59029G/A polymorphism and DN susceptibility; (3) offering sufficient information on genotype and/ or allele frequencies in case and control groups; and (4) using validated genotyping method. Publications were eliminated from the present meta-analysis if any one of the following situations appeared: (1) adopting duplicated data on study participants; (2) animals as study objects; and (3) commentaries, case reports, review articles or conference abstracts. As for reports containing the same group of participants, we selected the one with the largest sample size.

\section{Data extraction}

Data extraction was done independently by two investigators according to the same standard form. The major information included first author's name, publication year, original country, ethnicity, type of diabetes mellitus, number of cases and controls, genotyping method, genotype and/or allele frequencies in case and control groups, and $P$ value for Hardy-Weinberg equilibrium (HWE) in controls. If more than one study was incorporated in one single article, their data were abstracted separately.

\section{Quality analysis of included studies}

In this meta-analysis, quality assessment of included studies was conducted using the criteria of NewcastleOttawa Scale (NOS). 0-4 scores means the poor quality and the article with 5-9 scores was the good quality study.

\section{Statistical analysis}

STATA 12.0 software (Stata Corporation, College Station, TX, USA) was applied to complete all statistical analyses in this study. The strength of the relationship between CCR5 59029G/A polymorphism and DN susceptibility was evaluated through calculating pooled odds ratios (ORs) with their corresponding 95\% confidence intervals (95\% CIs). Between-study heterogeneity was detected with chi-square-based Q test, with $P<0.05$ as the significant level. When significant heterogeneity appeared $\left(P_{\text {heterogeneity }}<0.05\right)$, the randomeffects model was employed to calculate pooled ORs, otherwise, fixed-effects model was used. Sensitivity analysis was performed through sequential omission of each included study to observe alteration in pooled ORs so as to test the stability of the final results. Begg's funnel plot and Egger's regression test were both adopted for publication bias examination among included studies. If publication bias was detected, Duval and Tweedie nonparametric "trim and fill' method was used to adjust publication bias.

\section{CONFLICTS OF INTEREST}

The authors declare no conflicts of interest. 


\section{REFERENCES}

1. Lee AS, Lee YJ, Lee SM, Yoon JJ, Kim JS, Kang DG, Lee HS. An aqueous extract of Portulaca oleracea ameliorates diabetic nephropathy through suppression of renal fibrosis and inflammation in diabetic $\mathrm{db} / \mathrm{db}$ mice. Am J Chin Med. 2012; 40:495-510.

2. Tervaert TW, Mooyaart AL, Amann K, Cohen AH, Cook HT, Drachenberg CB, Ferrario F, Fogo AB, Haas M, de Heer E, Joh K, Noël LH, Radhakrishnan J, et al, and Renal Pathology Society. Pathologic classification of diabetic nephropathy. J Am Soc Nephrol. 2010; 21:556-563.

3. Al-Lawati JA, Panduranga P, Al-Shaikh HA, Morsi M, Mohsin N, Khandekar RB, Al-Lawati HJ, Bayoumi RA. Epidemiology of Diabetes Mellitus in Oman: Results from two decades of research. Sultan Qaboos Univ Med J. 2015; 15:e226-233.

4. Dronavalli S, Duka I, Bakris GL. The pathogenesis of diabetic nephropathy. Nat Clin Pract Endocrinol Metab. 2008; 4:444-452.

5. Reutens AT. Epidemiology of diabetic kidney disease. Med Clin North Am. 2013; 97:1-18.

6. Graves DT, Kayal RA. Diabetic complications and dysregulated innate immunity. Front Biosci. 2008; 13:1227-1239.

7. Forbes JM, Cooper ME. Mechanisms of diabetic complications. Physiol Rev. 2013; 93:137-188.

8. Navarro-Gonzalez JF, Mora-Fernandez C. The role of inflammatory cytokines in diabetic nephropathy. J Am Soc Nephrol. 2008; 19:433-442.

9. Fiorina P, Bassi R, Gremizzi C, Vergani A, Caldara R, Mello A, Del Maschio A, De Cobelli F, Perseghin G, Secchi A. 31P-magnetic resonance spectroscopy (31P-MRS) detects early changes in kidney high-energy phosphate metabolism during a 6-month Valsartan treatment in diabetic and nondiabetic kidney-transplanted patients. Acta Diabetol. 2012; 49:S133-139.

10. D'Addio F, Trevisani A, Ben Nasr M, Bassi R, El Essawy B, Abdi R, Secchi A, Fiorina P. Harnessing the immunological properties of stem cells as a therapeutic option for diabetic nephropathy. Acta Diabetol. 2014; 51:897-904.

11. Cavalera M, Frangogiannis NG. Targeting the chemokines in cardiac repair. Curr Pharm Des. 2014; 20:1971-1979.

12. Fox JM, Kasprowicz R, Hartley O, Signoret N. CCR5 susceptibility to ligand-mediated down-modulation differs between human T lymphocytes and myeloid cells. J Leukoc Biol. 2015; 98:59-71.

13. Flynn JK, Paukovics G, Moore MS, Ellett A, Gray LR, Duncan R, Salimi H, Jubb B, Westby M, Purcell DF, Lewin SR, Lee B, Churchill MJ, et al. The magnitude of HIV-1 resistance to the CCR5 antagonist maraviroc may impart a differential alteration in HIV-1 tropism for macrophages and T-cell subsets. Virology. 2013; 442:51-58.
14. Oo Z, Barrios CS, Castillo L, Beilke MA. High levels of CC-chemokine expression and downregulated levels of CCR5 during HIV-1/HTLV-1 and HIV-1/HTLV-2 coinfections. J Med Virol. 2015; 87:790-797.

15. Leach K, Charlton SJ, Strange PG. Analysis of second messenger pathways stimulated by different chemokines acting at the chemokine receptor CCR5. Biochem Pharmacol. 2007; 74:881-890.

16. Jin J, Colin P, Staropoli I, Lima-Fernandes E, Ferret C, Demir A, Rogée S, Hartley O, Randriamampita C, Scott MG, Marullo S, Sauvonnet N, Arenzana-Seisdedos F, et al. Targeting spare CC chemokine receptor 5 (CCR5) as a principle to inhibit HIV-1 entry. J Biol Chem. 2014; 289:19042-19052.

17. Al-Mahruqi SH, Zadjali F, Koh CY, Balkhair A, Said EA, Al-Balushi MS, Hasson SS, Al-Jabri AA. New genetic variants in the CCR5 gene and the distribution of known polymorphisms in Omani population. Int J Immunogenet. 2014; 41:20-28.

18. Li H, Liu TJ, Hong ZH. Gene polymorphisms in CCR5, CCR2, SDF1 and RANTES among Chinese Han population with HIV-1 infection. Infect Genet Evol. 2014; 24:99-104.

19. Ying H, Wang J, Gao X. CCL5-403, CCR5-59029, and Delta32 polymorphisms and cancer risk: a metaanalysis based on 20,625 subjects. Tumour Biol. 2014; 35:5895-5904.

20. Carpenter D, Taype C, Goulding J, Levin M, Eley B, Anderson S, Shaw MA, Armour JA. CCL3L1 copy number, CCR5 genotype and susceptibility to tuberculosis. BMC Med Genet. 2014; 15:5.

21. Dhaouadi T, Sfar I, Aounallah-Skhiri H, Jendoubi-Ayed S, Bouacha H, Ben Abdallah T, Gorgi Y. MCP-1, CCR2 and CCR5 polymorphisms in Tunisian patients with atopic asthma. Iran J Allergy Asthma Immunol. 2013; 12:29-36.

22. Ahluwalia TS, Khullar M, Ahuja M, Kohli HS, Bhansali A, Mohan V, Venkatesan R, Rai TS, Sud K, Singal PK. Common variants of inflammatory cytokine genes are associated with risk of nephropathy in type 2 diabetes among Asian Indians. PLoS One. 2009; 4:e5168.

23. Buraczynska M, Zukowski P, Wacinski P, Berger-Smyka B, Dragan M, Mozul S. Chemotactic cytokine receptor 5 gene polymorphism: relevance to microvascular complications in type 2 diabetes. Cytokine. 2012; 58:213-217.

24. Mlynarski WM, Placha GP, Wolkow PP, Bochenski JP, Warram JH, Krolewski AS. Risk of diabetic nephropathy in type 1 diabetes is associated with functional polymorphisms in RANTES receptor gene (CCR5): a sex-specific effect. Diabetes. 2005; 54:3331-3335.

25. Mokubo A, Tanaka Y, Nakajima K, Watada H, Hirose T, Kawasumi M, Sakai K, Kanazawa A, Maeda S, Hosokawa K, Atsumi Y, Matsuoka K, Kawamori R. Chemotactic cytokine receptor 5 (CCR5) gene promoter polymorphism $(59029 \mathrm{~A} / \mathrm{G})$ is associated with diabetic nephropathy in Japanese patients with type 2 diabetes: a 10-year longitudinal study. Diabetes Res Clin Pract. 2006; 73:89-94. 
26. Nakajima K, Tanaka Y, Nomiyama T, Ogihara T, Piao L, Sakai K, Onuma T, Kawamori R. Chemokine receptor genotype is associated with diabetic nephropathy in Japanese with type 2 diabetes. Diabetes. 2002; 51:238-242.

27. Nakajima K, Tanaka Y, Nomiyama T, Ogihara T, Ikeda F, Kanno R, Iwashita N, Sakai K, Watada H, Onuma T, Kawamori R. RANTES promoter genotype is associated with diabetic nephropathy in type 2 diabetic subjects. Diabetes Care. 2003; 26:892-898.

28. Pettigrew KA, McKnight AJ, Patterson CC, Kilner J, Sadlier DM, Maxwell AP. Resequencing of the CCL5 and CCR5 genes and investigation of variants for association with diabetic nephropathy. J Hum Genet. 2010; 55:248-251.

29. Prasad P, Tiwari AK, Kumar KM, Ammini AC, Gupta A, Gupta R, Thelma BK. Association of TGFbeta1, TNFalpha, CCR2 and CCR5 gene polymorphisms in type-2 diabetes and renal insufficiency among Asian Indians. BMC Med Genet. 2007; 8:20.

30. Yadav AK, Kumar V, Dutta P, Bhansali A, Jha V. Variations in CCR5, but not HFE, ELMO1, or SLC12A3, are associated with susceptibility to kidney disease in north Indian individuals with type 2 diabetes. J Diabetes. 2014; 6:547-555.
31. Cheng J, Liu RH, Liu FY, Peng YM. Influence of CCR5 genetic polymorphisms on diabetic nephropathy in Han population with type 2 diabetes in Hunan province. Shandong Medical Journal. 2010; 50:62-63.

32. Li HF, Song DP, Zhan M, Liu H, Wang YM, Duan Y. The polymorphism of promoter region 59029G/A of CCR5 gene is related to diabetic nephropathy. Chinese Journal of Diabetes. 2005; 13:206-208, 212.

33. Wang R, Zhang S, Shi LX. Association between polymorphism of CCR5 gene with diab etic nephropathy in type 2 diabetes mellitus in Chinese. Guizhou Medical Journal. 2007; 31:500-503.

34. Zhao YM, Li HF, Song DP. Correlativity Investigation Between the Variants in RANTES Gene of CCR5 and Its Ligand and the Diabetic Nephrosis. J Med Res. 2006; 35:45-46.

35. Thompson JR, Minelli C, Abrams KR, Tobin MD, Riley RD. Meta-analysis of genetic studies using Mendelian randomization--a multivariate approach. Stat Med. 2005; 24:2241-2254

36. Thakkinstian A, McElduff P, D'Este C, Duffy D, Attia J. A method for meta-analysis of molecular association studies. Stat Med. 2005; 24:1291-1306. 